\title{
Non-conventional fotemustine schedule as second-line treatment in recurrent malignant gliomas: Survival across disease and treatment subgroup analysis and review of the literature
}

\author{
ARSELA PRELAJ $^{1 *}$, SARA ELENA REBUZZI ${ }^{2 *}$, MASSIMILIANO GRASSI $^{2}$, MAURIZIO SALVATI $^{3}$, \\ ALESSANDRO D'ELIA ${ }^{3}$, FRANCESCA BUTTARELLI ${ }^{4}$, CARLA FERRARA $^{5}$, \\ SILVERIO TOMAO $^{1}$ and VINCENZO BIANCO ${ }^{1}$
}

\begin{abstract}
${ }^{1}$ Department of Radiological, Oncological and Anatomo-Pathological Sciences, 'Sapienza' University of Rome, Policlinico Umberto I, I-00161 Rome; ${ }^{2}$ Department of Medical Oncology, Ospedale Policlinico San Martino IST, University of Genoa, I-16132 Genoa; ${ }^{3}$ Neurosurgery Department, IRCCS NEUROMED INM, Neurochirurgia, I-86077 Pozzilli; ${ }^{4}$ Department of Neurology and Psychiatry 'Sapienza' University of Rome, Policlinico Umberto I, I-00161 Rome;

${ }^{5}$ Department of Public Health and Infectious Diseases, 'Sapienza' University of Rome, I-00185 Rome, Italy
\end{abstract}

Received March 5, 2018; Accepted July 26, 2018

DOI: $10.3892 / \mathrm{mco} .2018 .1746$

\begin{abstract}
Fotemustine (FTM) is a treatment option in recurrent malignant gliomas (MGs) after first-line Stupp treatment. The efficacy and the safety of fractionated FTM schedule proposed by Addeo et al was analysed in the present study in recurrent MGs patients. A retrospective analysis on 40 recurrent MGs patients and second-line fractionated FTM chemotherapy was performed. Response evaluation was assessed using RANO criteria and safety was assessed using CTCAE v.4.03. Subgroup analyses based on MGMT methylation, resurgery and reirradiation were performed. A review of the literature was also performed. The results revealed 5 partial responses $(13 \%)$ and 19 stable diseases $(47 \%)$ with a disease-control rate of $60 \%$. Median progression-free survival (PFS) was 4 months, with a PFS of $33 \%$ at 6 months and $13 \%$ at 1 year. The median overall survival (OS) was 9 months and OS at 6 months was of $55 \%$ and at 1 year of $30 \%$. Methylated patients experienced longer mPFS (6 vs. 3 months; $\mathrm{p}=0.004$ ) and mOS (10 vs. 4 months; $\mathrm{p}<0.0001$ ) compared with unmethylated patients. Patients treated with reirradiation experienced longer mPFS (5 vs. 3.5 months; $p=0.48$ ) and mOS (10 vs. 5 months; $\mathrm{p}=0.11$ ). No survival benefit with resurgery was observed. Furthermore, the fractioned schedule was well tolerated, only $15 \%$ of patients developed severe myelotoxicities. Considering the present findings, fractionated FTM
\end{abstract}

Correspondence to: Dr Arsela Prelaj, Department of Radiological, Oncological and Anatomo-Pathological Sciences, 'Sapienza' University of Rome, Policlinico Umberto I, Viale del Policlinico 155, I-00161 Rome, Italy

E-mail: arsela20@hotmail.it

*Contributed equally

Key words: recurrent glioblastoma, recurrent malignant glioma, fotemustine, second-line, MGMT, surgery, irradiation schedule is an efficient second-line option for MGs associated with an acceptable myelotoxicity profile. Additionally, MGMT methylation is associated with improved survival outcomes. However, this study highlights the requirement for further prospective randomized studies on resurgery and reirradiation.

\section{Introduction}

Malignant gliomas (MGs) are the most common primary malignant brain tumours in adults ( $\sim 60 \%$ of the total) (1). According to WHO classification of brain tumours, MGs include grade III gliomas (or anaplastic gliomas-AGs) and glioblastoma multiforme (GBM) $(2,3)$, which is the most frequent and aggressive $\mathrm{MG}$, characterised by a high recurrence and mortality rate. Standard first-line therapy of GBM includes maximal-safe surgical resection followed by radiotherapy plus concomitant and adjuvant temozolomide (TMZ), as defined in the EORTC Phase III trial (4). Despite the optimal standard treatment, recurrence rates are high $(\sim 90 \%)$ with median survival ranges from 15-18 months for GBM (5) and from 2-5 years for AGs (6).

On the contrary, there are no standard therapies for recurrent MGs. Different options are under investigation, including resurgery, reirradiation and chemotherapy, and their combinations (7).

Fotemustine (FTM) is a third-generation nitrosourea, an alkylating agent, which is a widely used and studied therapeutic option for MGs recurring after TMZ treatment, especially in Europe (8-11).

There are two types of FTM schedule. The standard schedule includes an induction phase dose of $100 \mathrm{mg} / \mathrm{mq}$ weekly for 3 consecutive weeks followed by a 5-week rest period and a maintenance phase dose of $100 \mathrm{mg} / \mathrm{mq}$ every 3 weeks (12-15). Haematological toxicity is observed in $>30 \%$ of patients $(9,16)$, mainly at the end of the induction phase, with grade 3-4 thrombocytopenia and neutropenia occurring in $\sim 14 \%$ of patients (11). 
In 2011 Addeo et al proposed a fractionated FTM schedule which includes an induction phase dose of $80 \mathrm{mg} / \mathrm{mq}$ every 2 weeks for 5 consecutive weeks followed by a 4-week rest period and a maintenance phase dose of $80 \mathrm{mg} / \mathrm{mq}$ every 4 weeks (16). This treatment is associated with a lower rate of myelotoxicities, with grade 3 thrombocytopenia and leukopenia occurring in only 7 and $3 \%$ of patients respectively $(10,16)$.

Resurgery and reirradiation are two controversial therapeutic options due to the lack of prospective randomized trials but multiple trials have studied their survival and clinical benefit especially in combination with subsequent chemotherapy $(7,17,18)$.

We retrospectively analysed recurrent MGs patients receiving fractionated FTM schedule proposed by Addeo et al (16) in order to evaluate the efficacy and safety of this second-line option according to disease and treatment subgroup analysis and compared to the literature.

\section{Patients and methods}

Study population. From the database of the Department of Oncology Unit A of Policlinico Umberto I, Sapienza University of Rome, we performed a retrospective analysis of consecutive recurrent MGs patients treated with Addeo FTM schedule after a first-line therapy with maximum safe resection and adjuvant TMZ-based therapy. We excluded those patients with evidence of cerebral haemorrhage on baseline MRI, treated with FTM standard schedule or FTM combined with other therapies or affected by clinically significant medical conditions that would make the treatment unsafe.

For all patients, the initial diagnosis was established by magnetic resonance imaging (MRI) and histologically using WHO criteria.

$\mathrm{O}^{6}$-methylguanine-DNA methyltransferase (MGMT) promoter methylation and IDH mutation analyses were performed (3) using a digital polymerase chain reaction (PCR) method. The recurrence after first-line therapy was established by MRI and histological examination when surgery was performed.

Clinical data at recurrence included patients characteristics (sex, age and Karnofsky performance status (KPS) at recurrence) tumour characteristics (laterality and lobe interested, histotype at recurrence, MGMT methylation and IDH mutation status at diagnosis) and treatment information (surgery and radiotherapy at recurrence, line of treatment and median cycles of FTM, first-line therapy, interval between completion of RT/TMZ and recurrence, next lines of chemotherapy and completion of induction phase).

Treatment plan. All patients included in the analysis underwent second-line treatment with FTM alone, according to the new Addeo schedule (16): induction phase dose of $80 \mathrm{mg} / \mathrm{mq}$ every 2 weeks for 5 consecutive weeks followed by a 4 -weeks rest period and a maintenance phase dose of $80 \mathrm{mg} / \mathrm{mq}$ every 4 weeks.

Response and toxicity evaluation. All patients were followed clinically by a multidisciplinary team and radiologically by MRI scans. The evaluations were made after the induction phase (5 cycles of chemotherapy) and then every 2 cycles during the maintenance phase or whenever progression disease was clinically suspected. Evaluation response was assessed according to RANO criteria (19) as complete (CR) and partial (PR) response, stable (SD) and progression (PD) disease. Disease control (DC) was defined as the sum of CR, PR and SD. Diagnosis of recurrence was determined by MRI in all patients and by histological examination when a second surgical resection was made.

All adverse events, as worsening of previous symptoms or development of new symptoms during treatment, were graded according to the Common Terminology Criteria for Adverse Events (CTCAE) of the National Cancer Institute, version 4.03 (20). Toxicity was evaluated at 2 -weekly intervals or, if clinically indicated, at weekly intervals.

Statistical analysis. Survival analysis was conducted on the efficacy of FTM in recurrent MGs in terms of 6 and 12 months progression-free survival (PFS) and overall survival (OS), median PFS (mPFS) and OS (mOS) from FTM treatment. Safety analysis evaluated the toxicity profile of FTM Addeo schedule compared to the standard schedule.

PFS was measured from the start of FTM therapy to diagnosis of PD evidenced by MRI or to death from any cause or to last follow-up assessment. OS was measured from the start of treatment with FTM to death from any cause or last follow-up.

Median PFS and OS were estimated with their 95\% confidence interval. Survival curves of PFS and OS were generated using the Kaplan-Meier method (21). Differences in PFS and OS were evaluated using the log-rank test (Mantel-Cox) for statistical significance, which was defined at the $\mathrm{p}<0.05$ level (21).

\section{Results}

Patients' characteristics. Between August 2010 and October 2017, 40 patients with recurrent MGs receiving FTM schedule proposed by Addeo et al as second-line treatment were included in the analysis. Patient, tumour and treatment characteristics at recurrence are summarised in Table I.

Most patients were male (68\%), the median age was 54 years (range 25-75 years) and median KPS was 80 (range, 60-90). All patients had a histological diagnosis of MGs: GBM was the predominant histotype (85\%), while grade III gliomas represented the $15 \%$ of the total. At first relapse all grade-III grade gliomas evolved in GBM (secondary GBM), whose diagnosis was made radiologically in 5 patients and histologically after resurgery in 1 patient.

The assessment of the MGMT promoter methylation status was conducted in 36 patients $(90 \%)$. MGMT promoter was methylated in 20 patients $(50 \%)$ and unmethylated in 16 patients $(40 \%)$. The assessment of the IDH mutation status was conducted in 25 patients $(68 \%)$. IDH gene was mutated in 5 patients $(13 \%)$ and non mutated in 22 patients $(55 \%)$.

All patients underwent surgery at diagnosis and were treated as first-line treatment with radiotherapy plus concomitant and adjuvant TMZ as Stupp protocol (4). At first recurrence, 63\% of patients underwent resurgery and $42 \%$ of patients were treated with radiotherapy.

All patients received FTM as second-line treatment according to Addeo schedule. Most patients (83\%) started 
Table I. Patient $(n=40)$ and tumor characteristics at recurrence.

\begin{tabular}{ll}
\hline Characteristics & $\mathrm{n}(\%)$ \\
\hline
\end{tabular}

Sex

Male

$27(68)$

Female

Median age, years (range)

Karnofsky performance status

Median (range)

90-100

$70-80$

60

Laterality

Right

Left

Histotype

Glioblastoma multiforme

Anaplastic glioma

MGMT promoter methylation status at diagnosis

Methylated

Unmethylated

Unknown

IDH mutation status at diagnosis

Mutated

Non mutated

Unknown

$13(32)$
$54(25-75)$
$80(60-90)$
$7(17)$
$28(70)$
$5(13)$

$22(55)$

18 (45)

$34(85)$

$6(15)$

$20(50)$

$16(40)$

4 (10)

$11(28)$

$16(40)$

13 (32)

Surgery at recurrence

Yes

25 (63)

No

$\mathrm{RT}$ at recurrence

Yes

17 (42)

No

Interval between completion

of RT/TMZ and recurrence

$\leq 3$ months

$33(83)$

No. of lines of chemotherapy after FTM

None

One

Two

In progress with FTM

Median cycles received, no. (range)

Induction phase completed

Yes

No

$24(60)$

11 (28)

$3(7)$

$5(2-50)$

27 (68)

$13(32)$

MGMT, $\mathrm{O}^{6}$-methylguanine-DNA-methyltransferase; IDH, isocitrate dehydrogenase; RT, radiotherapy; TMZ, temozolomide, FTM, fotemustine.

FTM after 3 months from TMZ last administration, in order to reduce the influence of pseudoprogression effect on the occurrence evaluation.
Table II. Results of FTM as second-line therapy in malignant gliomas.

\begin{tabular}{lccc} 
A, Objective responses, $\mathrm{n}(\%)$ & & \\
\hline & GBM & AG & Total patients \\
\hline Response & & & \\
Complete responses & $0(0)$ & $0(0)$ & $0(0)$ \\
Partial response & $3(9)$ & $2(33)$ & $5(13)$ \\
Stable disease & $17(50)$ & $2(33)$ & $19(47)$ \\
Progressive disease & $14(41)$ & $2(33)$ & $16(40)$ \\
Disease control & $20(59)$ & $4(67)$ & $24(60)$ \\
\hline
\end{tabular}

B, Survival data

\begin{tabular}{lc}
\hline Survival & Values \\
\hline 6 months-PFS, \% & 33 \\
12 months-PFS, \% & 13 \\
Median PFS, months (range) & $4(2-49)(3.02-5)$ \\
$(95 \%$ CI) & 55 \\
6 months-OS, \% & 30 \\
12 months-OS, \% & $9(2.5-50.5)(7.36-10.64)$ \\
Median OS, months (range) & 33 \\
(95\% CI) & \\
Complete responses, \% & \\
\hline
\end{tabular}

GBM, glioblastoma multiforme; AG, anaplastic glioma; PFS, progression-free survival; OS, overall survival; CI, confidence interval.

All patients received at least two doses of FTM. The median number of cycles administered was 5 (range, 2-50) with a completion of the induction phase in the $68 \%$ of patients. The main reason for not beginning maintenance phase was disease progression. Fourteen patients (35\%) with progressive disease after FTM underwent further lines of chemotherapy: 11 patients $(28 \%)$ underwent a third line chemotherapy and 3 patients (7\%) up to a fourth line.

Activity evaluation. All patients included in the study were assessable for response analysis (Table II). Among the 40 patients, 5 patients achieved PR (13\%) and 19 patients SD (47\%) with a DCR of $60 \%$.

The mPFS was 4 months (95\% CI 3.02-5) with a PFS-6 of $33 \%$ and a PFS at 1 year (PFS-1y) of $13 \%$ (Fig. 1A). The mOS from the start of FTM was 9 months (95\% CI 7.36-10.64) with an OS at 6 months of $55 \%$ and an OS at 1 year of $30 \%$ (Fig. 1B). Subgroup analyses were performed and are summarised in Table III.

Patients who completed the induction phase $(n=27)$ experienced longer survival outcomes with a mPFS of 6 vs. 3 months $(\mathrm{p}=0.02)$ and a mOS of 10 vs. 4 months $(\mathrm{p}=0.004)$. Patients with a KPS $>70 \%(70 \%)$ were associated with a higher OS (10 vs. 8 months) and a similar PFS (5 vs. 4 months) compared to patients with a lower KPS. 
Table III. Subgroup analyses.

\begin{tabular}{|c|c|c|c|c|c|c|c|}
\hline & No. of patients & mPFS & $95 \% \mathrm{CI}$ & P-value & $\mathrm{mOS}$ & $95 \% \mathrm{CI}$ & $\mathrm{p}$-value \\
\hline \multicolumn{8}{|c|}{ KPS, \% } \\
\hline$>70$ & 28 & 5.0 & $3.8-6.2$ & \multirow[t]{2}{*}{0.72} & 10 & $5.3-14.7$ & \multirow[t]{2}{*}{0.22} \\
\hline$<70$ & 12 & 4.0 & $0-10.8$ & & 8 & $3.8-12.2$ & \\
\hline \multicolumn{8}{|c|}{ MGMT methylation } \\
\hline Yes & 20 & 6.0 & $3.0-8.9$ & \multirow[t]{2}{*}{0.004} & 10 & $6.5-13.5$ & \multirow[t]{2}{*}{$<0.000$} \\
\hline No & 16 & 3.0 & $2.5-3.5$ & & 4 & $2.2-5.8$ & \\
\hline \multicolumn{8}{|c|}{ IDH mutation } \\
\hline Yes & 11 & 4.0 & $2.4-5.6$ & \multirow[t]{2}{*}{0.94} & 10 & 6.4-13.6 & \multirow[t]{2}{*}{0.91} \\
\hline No & 16 & 3.0 & $1.3-4.7$ & & 10 & $4.4-15.6$ & \\
\hline \multicolumn{8}{|c|}{ Induction phase completed } \\
\hline Yes & 27 & 6.0 & $4.1-7.9$ & \multirow[t]{2}{*}{0.02} & 10 & $8.8-11.2$ & \multirow[t]{2}{*}{0.004} \\
\hline No & 13 & 3.0 & $1.7-4.3$ & & 4 & $2.2-5.8$ & \\
\hline \multicolumn{8}{|c|}{$\mathrm{RT}$ ar recurrence } \\
\hline Yes & 17 & 5.0 & $2.9-7.1$ & \multirow[t]{2}{*}{0.48} & 10 & $3.7-6.3$ & \multirow[t]{2}{*}{0.11} \\
\hline No & 23 & 3.5 & $2.5-4.5$ & & 5 & 0.11 & \\
\hline \multicolumn{8}{|c|}{ Surgery at recurrence } \\
\hline Yes & 25 & 6.0 & $0.1-11.9$ & \multirow[t]{2}{*}{0.18} & 9 & 5.612 .4 & \multirow[t]{2}{*}{0.95} \\
\hline No & 15 & 5.0 & $3.9-6.1$ & & 9 & $2-16$ & \\
\hline
\end{tabular}

CI, confidence interval; mPFS, median progression-free survival; mOS, median overall survival; KPS, Karnofsky performance status; MGMT, $\mathrm{O}^{6}$-methylguanine-DNA methyltransferase; IDH, isocitrate dehydrogenase; RT, radiotherapy.

Table IV. Toxicities by grade of severity, according to the CTCAE (version 4.03).

\begin{tabular}{|c|c|c|c|c|}
\hline Chemotherapy-related toxicity & Grade $1, \mathrm{n}(\%)$ & Grade $2, \mathrm{n}(\%)$ & Grade 3, n (\%) & Grade $4, \mathrm{n}(\%)$ \\
\hline \multicolumn{5}{|l|}{ Haematological } \\
\hline Thrombocytopenia & $14(38)$ & $5(14)$ & $1(3)$ & $1(3)$ \\
\hline Leukopenia & $11(30)$ & $6(16)$ & $1(3)$ & $1(3)$ \\
\hline Neutropenia & $5(14)$ & $4(11)$ & $1(3)$ & - \\
\hline Lymphopenia & $3(8)$ & $4(11)$ & $3(8)$ & $1(3)$ \\
\hline Anemia & $14(38)$ & $3(8)$ & - & - \\
\hline \multicolumn{5}{|l|}{ Hypertransaminasemia } \\
\hline ALT & $9(24)$ & $1(3)$ & - & - \\
\hline AST & $1(3)$ & - & - & - \\
\hline$\gamma \mathrm{GT}$ & $4(11)$ & $2(5)$ & - & - \\
\hline \multicolumn{5}{|l|}{ Renal } \\
\hline Creatinine increase & $7(19)$ & - & $1(3)$ & - \\
\hline \multicolumn{5}{|l|}{ Gastrointestinal } \\
\hline Nausea & $5(14)$ & $2(5)$ & - & - \\
\hline Diarrhea & - & $1(3)$ & $1(3)$ & - \\
\hline Mucositis & $1(3)$ & - & $1(3)$ & - \\
\hline \multicolumn{5}{|l|}{ General } \\
\hline Astenia & $6(16)$ & $6(16)$ & $1(3)$ & - \\
\hline Anorexia & - & $2(5)$ & $6(16)$ & - \\
\hline
\end{tabular}

ALT, alanine aminotransferase; AST, aspartate aminotransferase; $\gamma \mathrm{GT}, \gamma$-glutamyl transferase.

and mOS (10 vs. 4 months) compared to unmethylated patients $(n=16)$ with statistical significance $(p=0.004$ and $p<0.0001$ 

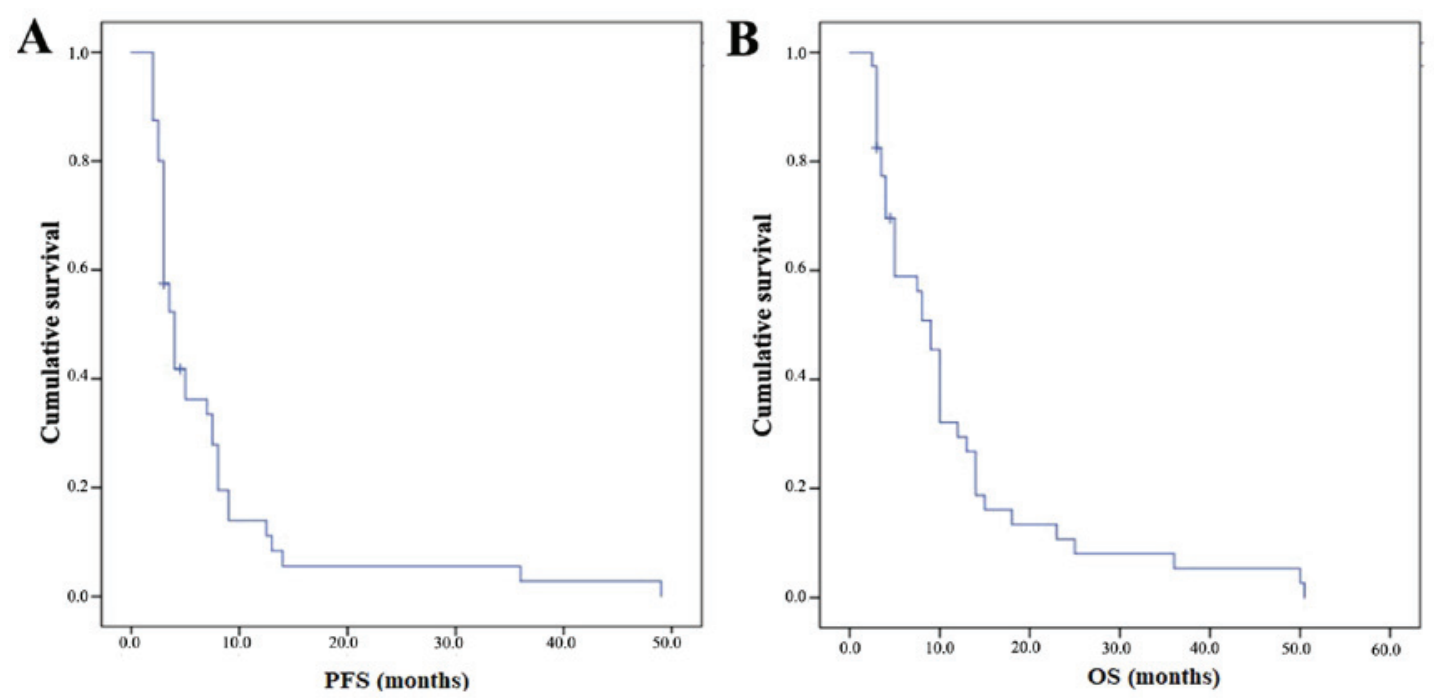

Figure 1. Kaplan-Meier survival curves of (A) PFS and (B) OS from the start of FTM treatment of the patients analysed in the study. PFS, progression-free survival; OS, overall survival; FTM, fotemustine.
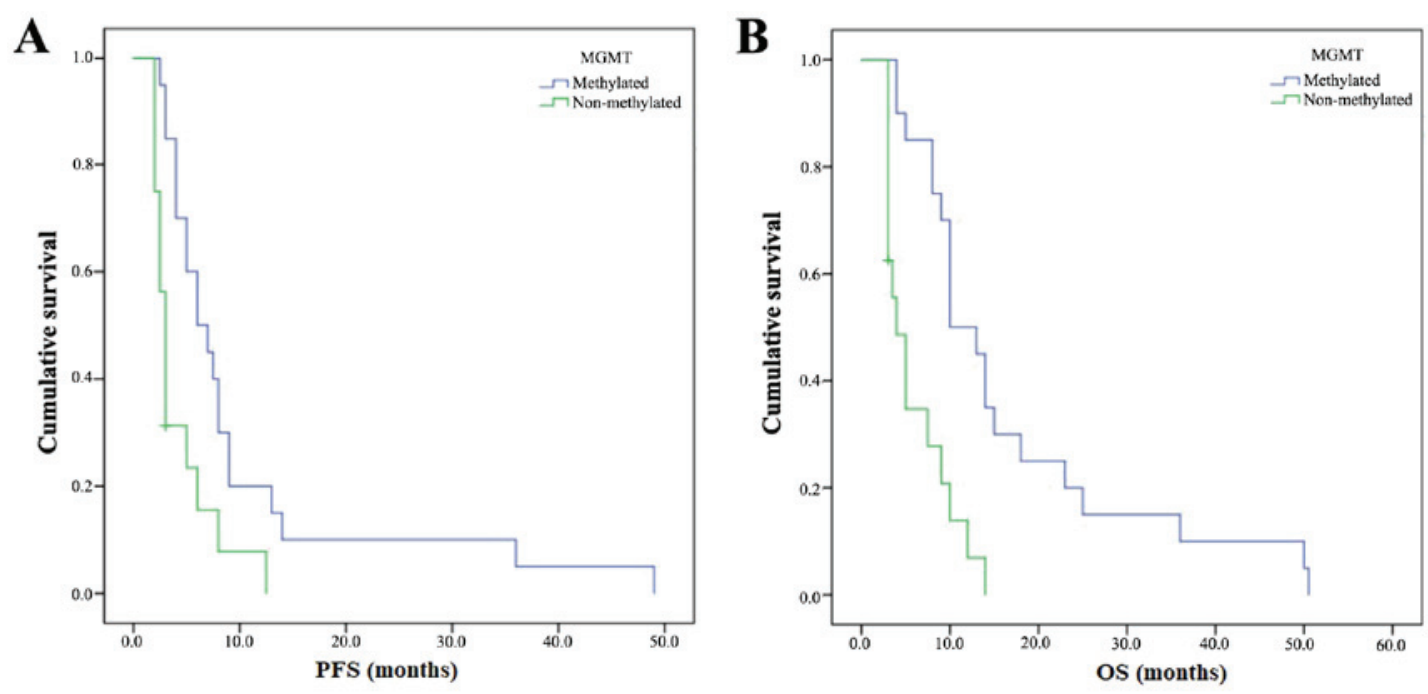

Figure 2. Kaplan-Meier survival curves of (A) PFS and (B) OS from the start of FTM treatment according to MGMT methylation. PFS, progression-free survival; OS, overall survival; FTM, fotemustine.

respectively) (Fig. 2B). Subgroup analyses according to IDH mutation were not performed due to the low number of patients.

Activity according to resurgery and reirradiation. Patients treated with radiotherapy at recurrence $(\mathrm{n}=17)$ before FTM therapy experienced longer mPFS (5 vs. 3.5 months) and longer mOS (10 vs. 5 months) without statistical significance $(\mathrm{p}=0.48$ and $\mathrm{p}=0.11$ respectively).

Patients who underwent surgery at recurrent $(n=25)$ before FTM therapy had similar mPFS (6 vs. 5 months) and mOS (both 9 months) compared to patients who did not undergo resurgery.

Toxicity evaluation. All 43 patients were evaluated for safety (Table IV). Grade 3-4 haematological toxicities were developed in 15\% of patients. Grade 3-4 thrombocytopenia and leukopenia occurred respectively in $6 \%$ of patients each. Grade 3 neutropenia was observed in $3 \%$ of patients. None of the patients developed grade 3-4 anaemia. No grade 3-4 non-haematological toxicities were recorded. The haematological toxicity incidence has a peak in correspondence of the induction completion, while during maintenance phase a higher incidence of hepatic, renal and general toxicity was observed. FTM was generally well tolerated and treatment interruption or death related to chemotherapy toxicity were not documented.

\section{Discussion}

Radiotherapy plus concomitant and adjuvant TMZ has become the standard treatment for newly diagnosed MGs with an improvement in OS and PFS compared to radiotherapy alone (4). However, recurrence probability is high ( $90 \%)$ and there is no consensus on second-line therapy, with treatment recommendations based on non-controlled phase II trials.

Nitrosoureas are the most widely studied therapeutic option, particularly FTM, a third generation nitrosourea. The use and 


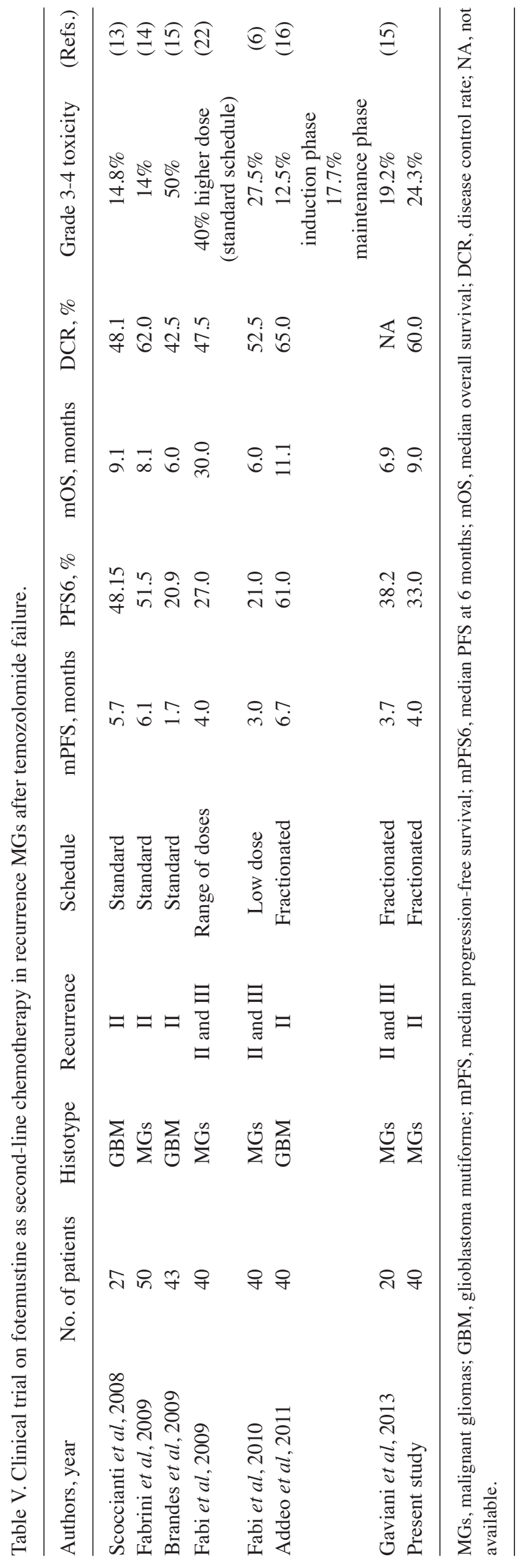


efficacy of FTM as second-line treatment in recurrent MGs are known especially in the conventional schedule proposed by Frenay et al (tree weekly FTM cycles at $100 \mathrm{mg} / \mathrm{mq}$ followed by a 5 -week rest and a maintenance phase with $100 \mathrm{mg} / \mathrm{mq}$ of FTM every three weeks) (12).

Several Italian phase II trials $(6,13-15,22)$ have investigated the use of FTM conventional schedule in MGs patients recurring after Stupp standard treatment with a wide range of survival data (Table V).

Scoccianti et al (13) and Fabrini et al (14) reported similar survival results in terms of PFS-6 rates $(\sim 50 \%)$, mPFS (nearly 6 months), mOS (nearly 8.6 months), DCR ( $55 \%)$ and grade 3-4 haematological toxicities $(14 \%)$ in 27 GBM and 50 MGs patients respectively.

Brandes et al (15) reported a mPFS and OS of 1.7 and 6 months respectively with a 6-PFS of 20,9\% and a DRC of $42,5 \%$. Haematological toxicity was high with grade 3-4 adverse events in nearly $50 \%$ of patients. This was the first study that showed a higher DCR in methylated MGMT patients (75\%) than in unmethylated MGMT patients $(34,6 \%)$ without statistical difference in terms of PFS-6 and OS between the two groups. Moreover, Brandes et al reported a significantly higher PFS- 6 in patients who started FTM $\geq 3$ months after TMZ completion than in those who started FTM within 3 months.

FTM standard regimen showed to be efficacy in recurrent MGs but was limited by considerable myelotoxicity, especially thrombocytopenia and leukopenia, with grade 3-4 adverse events in nearly $40 \%$ of patients. For this reason, subsequent phase II trials studied alternative FTM schedules in order to improve the safety profile of FTM second-line therapy.

In 2009 Fabi et al (22) analysed the activity of different doses of FTM, ranging from 65 to $100 \mathrm{mg} / \mathrm{mq}$, as second- or third-line therapy in $40 \mathrm{MGs}$ patients. The survival data were comparable to those of the conventional schedule, but with grade 3-4 haematological toxicities (40\% of patients) occurred only in the group with the highest FTM dosage. Fabi et al. confirmed that methylated MGMT patients went better than unmethylated MGMT patients in terms of PFS, OS and DCR, although this did not reach statistical significance.

In another prospective study (6), the same authors confirmed that lower doses of FTM $(60 \mathrm{mg} / \mathrm{mq}$ for the induction phase and $75 \mathrm{mg} / \mathrm{mq}$ for the maintenance phase) had the same activity of the full dose regimen but with a significantly lower rate of severe myelotoxicity $(27.5 \%)$.

To explain the wide range of PFS- 6 reported by these phase II trials (Table V), in 2012 Paccapelo et al (23) investigated different response pattern to FTM in correlation to the end of radiotherapy or predictive risk factors. They confirm that a longer time to progression after radiotherapy and surgery at recurrence are associated with a higher PFS-6 and DCR.

In 2011 Addeo et al (16) planned a new FTM schedule that maintains the global dosage but with different fractionation. Forty recurrent GBM patients received $80 \mathrm{mg} / \mathrm{mq}$ every 2 weeks for five consecutive administrations (induction phase) and then every 4 weeks at $80 \mathrm{mg} / \mathrm{mq}$ as maintenance, after a 4-week rest period. Efficacy data were similar to those of the conventional FTM schedule (mPFS of 6.7 months, PFS-6 of $61 \%$ and mOS of 11.1 months), but with a better DCR $(65 \%)$ and a lower rate of grade 3-4 myelotoxicity in the induction and maintenance phase $(12,50 \%$ and $17,70 \%$ respectively). They found also a trend toward prolonged PFS-6, without statistical relevance, and a higher DCR in methylated MGMT patients.

Only one prospective trial, performed by Gaviani et al (24), studied the Addeo FTM schedule at first or second recurrence. Survival data were quite comparable to other studies on standard FTM schedule (mPFS of 16 weeks with a 6-PFS of $38.2 \%$ and mOS of 30 weeks). The myelotoxicity profile was similar to that one of Addeo et al with a low rate of grade 3-4 thrombocytopenia and leukopenia ( $0.3 \%$ each), but with a higher rate of grade 3-4 lymphopenia (18.5\%).

In the present retrospective study, we analysed the efficacy and safety profile of fractionated FTM schedule proposed by Addeo et al as second-line therapy in 40 recurrent MGs patients. Survival analysis showed DCR of $60 \%$, mPFS of 4 months with PFS-6 of 33\% and mOS of 9 months with OS-6 of $55 \%$. These results are comparable to those reported by all the other trials on FTM, as reported in Table V. As concern Scoccianti, Fabrini and Addeo results, the favourable survival data, especially PFS, could be, in part, due to the inclusion in the study of patients with pseudoprogression rather than disease recurrence, with an overestimate of the results $(6,9,16)$. Pseudoprogression, a treatment-related reaction which mimics tumour progression, occurs mainly within 3 months after concurrent radio-chemotherapy with TMZ and correlates with favourable prognosis and higher response rate to $\operatorname{FTM}(25,26)$.

Differently from Brandes et al (15), Fabi et al $(6,22)$ and Addeo et al (16) we observed a statistical significance in terms of survival outcomes between methylated and unmethylated MGMT patients. The MGMT methylation status is prognostic at the time of diagnosis (27) but also in recurrent GBM as reported by the DIRECTOR and the BELOB trials $(28,29)$. The AVAREG trial (30) demonstrated also that MGMT methylation status was predictive of efficacy of FTM in the recurrence setting.

Subgroup analysis according to resurgery and reirradiation before FTM second-line treatment were performed since the evaluation of different treatment options and of their combinations is of high interest $(31,32)$ in order to improve local control of the disease and survival outcomes. Unfortunately, we didn't observe a survival benefit with resurgery probably due to the low number of patients, while data on reirradiation were more promising. Recent literature reviews and several retrospective studies showed a survival benefit with resurgery and reirradiation at the time of recurrence, with higher survival in selected patients with favourable clinical and radiological characteristics $(17,18)$.

Our results on grade 3-4 myelotoxicity (15\%) are comparable to those reported by Addeo et al and Gaviani et al $(16,24)$. Lower toxicity permits to preserve quality of life (QoL), which is an essential aim for MGs patients who can only be palliated (16). In fact, it is important to notice the high percentage of patients still fit for third (28\%) and fourth (7\%) line therapy after FTM failure (Table I).

Even if this is a retrospective analysis with a limited number of patients, a heterogeneous population (including primary and secondary GBM), our survival data are comparable to those presented by Addeo et al (16) and other phase II trials on standard schedule, with results which confirms that the fractionated FTM schedule has a better safety profile than standard regimen (Table V). 
From our experience, despite it remains unclear if the alternative schedule proposed by Addeo et al could be more effective than the standard one, we registered a low toxicity profile.

Considering these data, this fractionated FTM schedule can be an efficient second-line therapy in the treatment of such poor prognosis disease as it is MG, associated with less myelotoxicity compared to the literature.

Future phase III randomised trial with a larger number of patients and an adequate assessment of MGMT status at diagnosis and recurrence are needed to define the role of MGMT status in second-line therapy in MGs patients.

\section{Acknowledgements}

Not applicable.

\section{Funding}

No funding was received.

\section{Availability of data and materials}

The datasets used and/or analyzed during the current study are available from the corresponding author on reasonable request.

\section{Authors' contributions}

AP and SER contributed equally to this work and were the major contributors in writing the manuscript, analysing and interpreting the patient data. MG, MS, ADE, FB and CF were involved in acquisition, analysis and interpretation of patient data. ST and VB were involved in designing the study, writing the manuscript and revising it critically for important intellectual content. All authors read and approved the final manuscript.

\section{Ethics approval and consent to participate}

Not applicable.

\section{Patient consent for publication}

Not applicable.

\section{Competing interests}

The authors declare that they have no competing interests.

\section{References}

1. Ostrom Q, Gittleman H, Farah P, Ondracek A, Chen Y, Wolinsky Y, et al: CBTRUS statistical report: primary brain and central nervous system tumors diagnosed in the United States in 2006-2010. Neuro-Oncol 15: ii1-ii56, 2013.

2. Stupp R, Brada M, van den Bent MJ,Tonn JC and Pentheroudakis G; ESMO Guidelines Working Group: High-grade glioma: ESMO Clinical Practice Guidelines for diagnosis, treatment and follow-up. Ann Oncol 25 (Suppl 3): iii93-iii101, 2014.

3. Louis DN, Ohgaki H, Wiestler OD, Cavenee WK, Burger PC, Jouvet A, Scheithauer BW and Kleihues P: The 2007 WHO classification of tumours of the central nervous system. Acta Neuropathol 114: 97-109, 2007.
4. Stupp R, Mason WP, van den Bent MJ, Weller M, Fisher B, Taphoorn MJ, Belanger K, Brandes AA, Marosi C, Bogdahn U, et al; European Organisation for Research and Treatment of Cancer Brain Tumor and Radiotherapy Groups; National Cancer Institute of Canada Clinical Trials Group: Radiotherapy plus concomitant and adjuvant temozolomide for glioblastoma. $\mathrm{N}$ Engl J Med 352: 987-996, 2005.

5. Weller M, Cloughesy T, Perry JR and Wick W: Standards of care for treatment of recurrent glioblastoma-are we there yet? Neuro-oncol 15: 4-27, 2013.

6. Fabi A, Metro G, Vidiri A, Lanzetta G, Carosi M, Telera S, Maschio M, Russillo M, Sperduti I, Carapella CM, et al: Low-dose fotemustine for recurrent malignant glioma: A multicenter phase II study. J Neurooncol 100: 209-215, 2010.

7. Tosoni A, Franceschi E, Poggi R and Brandes AA: Relapsed Glioblastoma: Treatment Strategies for Initial and Subsequent Recurrences. Curr Treat Options Oncol 17: 49, 2016.

8. Beauchesne P: Fotemustine: A third-generation nitrosourea for the treatment of recurrent malignant gliomas. Cancers (Basel) 4 : 77-87, 2012.

9. Silvani A, Gaviani P, Lamperti E, Botturi A, Ferrari D, Simonetti G and Salmaggi A: Lecture: Fotemustine in brain tumors. Neurol Sci 32 (Suppl 2): S255-S257, 2011.

10. Lombardi G, Farina P, Della Puppa A, Cecchin D, Pambuku A, Bellu L and Zagonel V: An overview of fotemustine in high-grade gliomas: From single agent to association with bevacizumab. BioMed Res Int 2014: 698542, 2014.

11. Kyritsis AP and Levin VA: An algorithm for chemotherapy treatment of recurrent glioma patients after temozolomide failure in the general oncology setting. Cancer Chemother Pharmacol 67: 971-983, 2011.

12. Frenay M, Giroux B, Khoury S, Derlon JM and Namer M: Phase II study of fotemustine in recurrent supratentorial malignant gliomas. Eur J Cancer 27: 852-856, 1991.

13. Scoccianti S, Detti B, Sardaro A, Iannalfi A, Meattini I, Leonulli BG, Borghesi S, Martinelli F, Bordi L, Ammannati F, et al: Second-line chemotherapy with fotemustine in temozolomide-pretreated patients with relapsing glioblastoma: A single institution experience. Anticancer Drugs 19: 613-620, 2008.

14. Fabrini MG, Silvano G, Lolli I, Perrone F, Marsella A, Scotti V and Cionini L: A multi-institutional phase II study on second-line Fotemustine chemotherapy in recurrent glioblastoma. J Neurooncol 92: 79-86, 2009.

15. Brandes AA, Tosoni A, Franceschi E, Blatt V, Santoro A Faedi M, Amistà P, Gardiman M, Labianca R, Bianchini C, et al: Fotemustine as second-line treatment for recurrent or progressive glioblastoma after concomitant and/or adjuvant temozolomide: A phase II trial of Gruppo Italiano Cooperativo di Neuro-Oncologia (GICNO). Cancer Chemother Pharmacol 64: 769-775, 2009.

16. Addeo R, Caraglia M, De Santi MS, Montella L, Abbruzzese A, Parlato C, Vincenzi B, Carraturo M, Faiola V, Genovese M, et al: A new schedule of fotemustine in temozolomide-pretreated patients with relapsing glioblastoma. J Neurooncol 102: 417-424, 2011.

17. Montemurro N, Perrini P, Blanco MO and Vannozzi R: Second surgery for recurrent glioblastoma: A concise overview of the current literature. Clin Neurol Neurosurg 142: 60-64, 2016.

18. Sulman EP, Ismaila N, Armstrong TS, Tsien C, Batchelor TT, Cloughesy T, Galanis E, Gilbert M, Gondi V, Lovely M, et al: Radiation Therapy for Glioblastoma: American Society of Clinical Oncology Clinical Practice Guideline Endorsement of the American Society for Radiation Oncology Guideline. J Clin Oncol 35: 361-369, 2017.

19. Wen PY, Macdonald DR, Reardon DA, Cloughesy TF, Sorensen AG, Galanis E, Degroot J, Wick W, Gilbert MR, Lassman $\mathrm{AB}$, et al: Updated response assessment criteria for high-grade gliomas: Response assessment in neuro-oncology working group. J Clin Oncol 28: 1963-1972, 2010.

20. Common Terminology Criteria for Adverse Events v4.03 (CTCAE). Available at: http://ctep.cancer.gov. Accessed August 10, 2017.

21. Kaplan E and Meier P: Nonparametric estimation from incomplete observations. J Am Stat Assoc 53: 457-481, 1958.

22. Fabi A, Metro G, Russillo M, Vidiri A, Carapella CM, Maschio M, Cognetti F, Jandolo B, Mirri MA, Sperduti I, et al: Treatment of recurrent malignant gliomas with fotemustine monotherapy: Impact of dose and correlation with MGMT promoter methylation. BMC Cancer 9: 101, 2009. 
23. Paccapelo A, Lolli I, Fabrini MG, Silvano G, Detti B, Perrone F, Savio G, Santoni M, Bonizzoni E, Perrone T, et al: A retrospective pooled analysis of response patterns and risk factors in recurrent malignant glioma patients receiving a nitrosourea-based chemotherapy. J Transl Med 10: 90, 2012.

24. Gaviani P, Simonetti G, Salmaggi A, Lamperti E and Silvani A Safety of second-line chemotherapy with non-conventional fotemustine schedule in recurrent high grade gliomas: A single institution experience. J Neurooncol 113: 527-529, 2013.

25. Brandes AA, Franceschi E, Tosoni A, Blatt V, Pession A, Tallini G, Bertorelle R, Bartolini S, Calbucci F, Andreoli A, et al: MGMT promoter methylation status can predict the incidence and outcome of pseudoprogression after concomitant radiochemotherapy in newly diagnosed glioblastoma patients. J Clin Oncol 26: 2192-2197, 2008.

26. Taal W, Brandsma D, de Bruin HG, Bromberg JE, Swaak-Kragten AT, Smitt PA, van Es CA and van den Bent MJ: Incidence of early pseudo-progression in a cohort of malignant glioma patients treated with chemoirradiation with temozolomide. Cancer 113: 405-410, 2008.

27. Hegi ME, Diserens AC, Gorlia T, Hamou MF, de Tribolet N, Weller M, Kros JM, Hainfellner JA, Mason W, Mariani L, et al: MGMT gene silencing and benefit from temozolomide in glioblastoma. N Engl J Med 352: 997-1003, 2005.

28. Weller M, Tabatabai G, Kästner B, Felsberg J, Steinbach JP, Wick A, Schnell O, Hau P, Herrlinger U, Sabel MC, et al; DIRECTOR Study Group: MGMT promoter methylation is a strong prognostic biomarker for benefit from dose-intensified temozolomide rechallenge in progressive glioblastoma: The DIRECTOR Trial. Clin Cancer Res 21: 2057-2064, 2015.
29. Taal W, Oosterkamp HM, Walenkamp AM, Dubbink HJ, Beerepoot LV, Hanse MC, Buter J, Honkoop AH, Boerman D, de Vos FY, et al: Single-agent bevacizumab or lomustine versus a combination of bevacizumab plus lomustine in patients with recurrent glioblastoma (BELOB trial): A randomised controlled phase 2 trial. Lancet Oncol 15: 943-953, 2014.

30. Brandes AA, Finocchiaro G, Zagonel V, Reni M, Caserta C, Fabi A, Clavarezza M, Maiello E, Eoli M, Lombardi G, et al: AVAREG: A phase II, randomized, noncomparative study of fotemustine or bevacizumab for patients with recurrent glioblastoma. Neuro-oncol 18: 1304-1312, 2016.

31. Azoulay M, Santos F, Shenouda G, Petrecca K, Oweida A, Guiot MC, Owen S, Panet-Raymond V, Souhami L and Abdulkarim BS: Benefit of re-operation and salvage therapies for recurrent glioblastoma multiforme: Results from a single institution. J Neurooncol 132: 419-426, 2017.

32. Seystahl K, Wick W and Weller M: Therapeutic options in recurrent glioblastoma-An update. Crit Rev Oncol Hematol 99: 389-408, 2016.

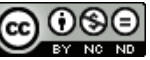

This work is licensed under a Creative Commons Attribution-NonCommercial-NoDerivatives 4.0 International (CC BY-NC-ND 4.0) License. 